OPEN ACCESS

Edited by:

Jianxun Ding,

Changchun Institute of Applied

Chemistry (CAS), China

Reviewed by:

Nenad Filipovic,

University of Kragujevac, Serbia

Qinghua Xu,

Purdue University, United States

*Correspondence:

Yukuan Qiu

qiuyukuan@163.com

Specialty section:

This article was submitted to

Biomaterials,

a section of the journal

Frontiers in Materials

Received: 14 October 2021 Accepted: 16 November 2021 Published: 13 December 2021

Citation:

Yang $Y$, Zhang $Q, X u Y$, Chen $G$ and Qiu Y (2021) Sulfated Polysaccharide From Undaria Pinnatifida Induces Apoptosis and Inhibits Proliferation, Migration, and Invasion in Ovarian Cancer via Suppressing the Hedgehog Signaling Pathway.

Front. Mater. 8:795061. doi: 10.3389/fmats.2021.795061

\section{Sulfated Polysaccharide From Undaria Pinnatifida Induces Apoptosis and Inhibits Proliferation, Migration, and Invasion in Ovarian Cancer via Suppressing the Hedgehog Signaling Pathway}

\author{
Yi Yang, Qin Zhang, Yongping Xu, Gang Chen and Yukuan Qiu* \\ Department of Oncology, China Coast Guard Hospital of the People's Armed Police Force, Jiaxing, China
}

Objective: To investigate the effects of sulfured polysaccharide from Undaria pinnatifida (SPUP) on the biological behaviors of ovarian cancer $(\mathrm{OC})$ cells and its potential mechanism.

Methods: Sulfated polysaccharide from Undaria pinnatifida (SPUP) was extracted and characterized through a combination of chemical analysis, IR spectra, UV-Vis, gas chromatography, and high-performance gel permeation chromatography. OC and human ovarian surface epithelial cells were used as working model in vitro for evaluation of SPUP's therapeutic effects. A combination of CCK-8, Transwell, and flow cytometry assay was used to measure the proliferation, migration, invasion, and apoptosis of $\mathrm{OC}$ cells, respectively. In addition, the protein expression levels of cells were also measured by Western blot.

Results: SPUP suppressed OC development from three different perspectives: 1) SPUP treatment significantly inhibited the proliferation of $\mathrm{OC}$ in a dosage-dependent manner $(p<$ 0.05); 2) SPUP inhibited the migration and invasion of OC cells confirmed by scratch and Transwell experiments $(p<0.05)$; 3) SPUP induced apoptosis in OC cells and thus further inhibited the growth of OC cells evaluated using flow cytometry $(p<0.05)$. The underlying mechanism of the suppressing effects of SPUP might be related to the inhibition of the hedgehog $(\mathrm{Hh})$ signaling pathway in OC cells after SPUP treatment. With additional suppression of the $\mathrm{Hh}$ signaling pathway, the anticancer effects of SPUP were enhanced $(p<0.05)$.

Conclusion: Taken together, SPUP could inhibit the proliferation, migration, and invasion and induce apoptosis of $\mathrm{OC}$ cells by inhibiting the activation of the Hh signaling pathway, which proposes SPUP as a novel drug to treat OC clinically.

Keywords: sulfated polysaccharide from undaria pinnatifida, epithelial ovarian cancer, hedgehog signaling pathway, apoptosis, proliferation, migration, invasion 


\section{INTRODUCTION}

There are approximately 239,000 new cases of ovarian cancer (OC), the deadliest gynecological tumor in women, developed and 152,000 deaths worldwide every year (Ferlay et al., 2015). Due to the difficulty of early diagnosis and the drug resistance of chemotherapy, OC has become the leading cause of death in patients with gynecological malignant tumors (Torre et al., 2018), among which epithelial OC is the most critical pathological subtype of OC, accounting for $90 \%$ of primary ovarian malignant tumors (Mutch and Prat, 2014; Fu et al., 2016). Till now, cytoreductive surgery combined with platinum and paclitaxel-based chemotherapy is the standard treatment strategy for epithelial OC. However, about $70 \%$ of patients relapse after the initial treatment and often develop resistance to platinum chemotherapy after recurrence (Chien et al., 2013). OC can metastasize rapidly in a short time and has a high degree of malignancy. Due to the fact that there is no satisfactory treatment for advanced patients, the 5-year survival rate of patients with advanced epithelial OC is only $29 \%$ (Reid et al., 2017). Therefore, it is urgent to develop a new, safe, and effective drug with minor side effects to control the progression of OC and reduce patient mortality.

In recent years, natural antitumor polysaccharides have gradually attracted the attention of cancer experts and scholars because of their significant anticancer effects with less toxic and side effects (Zong et al., 2015). It has been reported that polysaccharides could inhibit the proliferation of tumor cells and directly induce apoptosis or trigger immune enhancement activity combined with chemotherapy (Zong et al., 2012; Chowdhury et al., 2015). Undaria pinnatifida (UP), as seafood, is distributed worldwide (Zhou et al., 2015). The main compounds of UP are protein, polysaccharides, and minerals. Further, studies reported that UP had various biological functions, including immune regulation and anticancer (Wu et al., 2019; Yu et al., 2019). Sulfated polysaccharide from Undaria pinnatifida (SPUP), a critical component of US, is a hot research topic especially in the field of cancer treatment. It has been proved that SPUP plays a critical role in anti-breast cancer treatment by inhibiting the proliferation and migration of as well as inducing apoptosis in breast cancer cells (Wu et al., 2019). In addition, other studies have shown that SPUP could be encapsulated into nanoparticles and delivered through injection to inhibit the migration and invasion of pancreatic cancer cells; thus, SPUP was identified as a target drug for pancreatic cancer treatment (Song et al., 2019). However, the effects and activity of SPUP in OC remain unclear and require further studies.

The hedgehog $(\mathrm{Hh})$ signaling pathway plays an important role in determining cell fate including regulating proliferation, migration, and invasion (Liu et al., 2018). It was reported that the aberrant activation of the $\mathrm{Hh}$ signaling pathway was associated with tumorigenesis and progression of human cancer (Skoda et al., 2018). In OC, Liu et al. found that DHA could induce apoptosis and inhibit proliferation, migration, and invasion in ovarian cancer cell via suppressing the Hh signaling pathway (Liu et al., 2018). However, there is no study about the effects of SPUP on OC progression.

Taken together, this study aimed to study the effects of SPUP on cell proliferation, migration, invasion, and apoptosis in OC epithelial cells and further investigate its potential mechanism, providing novel insights for further potential identification of SPUP as a therapeutic drug in OC treatment.

\section{MATERIALS AND METHODS}

\section{Extraction and Identification of SPUP}

SPUP was prepared as previously published (Zhao et al., 2018). The UP was purchased from the local seafood market and rinsed with precooled ultrapure water to remove impurities. The cleaned UP was cut into 3-5-cm-long pieces and crushed with a highspeed grinder. The obtained homogenate was continuously stirred in absolute ethanol to remove lipids and other impurities. The suspension was centrifuged at room temperature at $5,000 \mathrm{rpm}$ for $30 \mathrm{~min}$ and air-dried to obtain powder. A volume of $20 \mathrm{~g}$ UP powder was resuspended in distilled water with a ratio of 1:20 (powder mass and water volume, $\mathrm{g} / \mathrm{mL}$ ), extracted for $5 \mathrm{~h}$ in the reflux device, and centrifuged at room temperature at 5,000 rpm for $20 \mathrm{~min}$. The supernatant was collected and concentrated to about $50 \mathrm{ml}$ with a vacuum rotary evaporator. It was then deproteinized by the Sevag method (Sevag et al., 1938). After that, the solution was mixed with four times the volume of absolute ethanol and stirred violently and overnight at $4^{\circ} \mathrm{C}$. The next day, the mixture was centrifuged at $4^{\circ} \mathrm{C}$ at $5,000 \mathrm{rpm}$ for $20 \mathrm{~min}$. The sediment was collected following by vacuuming and stored in the refrigerator at $-80^{\circ} \mathrm{C}$. To identify the properties of SPUP, chemical analysis, gas chromatography (GC), high-performance gel permeation chromatography (HPGPC), infrared spectroscopy, and ultraviolet, visible spectroscopy (UV-1800 spectrophotometer, Shimadzu, Kyoto, Japan) were used for evaluation.

\section{Cell Culture}

OC cell lines (SKOV3, A2780) and human ovarian surface epithelial cells (HOSEPICs) were purchased from Shanghai Fuheng Biotechnology Co., Ltd. The cells were incubated in a humidified incubator at $37^{\circ} \mathrm{C}$ and $5 \% \mathrm{CO}_{2}$ using RPMI 1640 medium with $10 \%$ fetal bovine serum and $1 \%$ penicillin and streptomycin.

\section{Cell Proliferation was Measured by Cell Counting Kit-8}

OC cells in the logarithmic growth stage were inoculated into 96well plates $\left(10^{4}\right.$ cells/well $)$ and cultured in an incubator at $37^{\circ} \mathrm{C}$ and $5 \% \mathrm{CO}_{2}$ for $24 \mathrm{~h}$. The cells were divided into the control and different-concentration SPUP treatment groups (the concentrations were $25,50,100,150$, and $200 \mu \mathrm{g} / \mathrm{ml})$, incubated for $24 \mathrm{~h}$ after corresponding treatment. After addition of $10 \mu \mathrm{l}$ CCK- 8 solution, the cells were cultured for another $2 \mathrm{~h}$ in the incubator. The 96-well plates were placed in a 
spectrophotometer to measure the absorbance value at $450-\mathrm{nm}$ wavelength.

\section{Cell Migration Activity was Detected by the Transwell Method}

The cells were divided into the control and SPUP treatment groups $(100 \mu \mathrm{g} / \mathrm{ml})$ and inoculated into the Transwell upper chamber (Corning, NY, USA) at the density of $10^{5}$ cells/well. The corresponding culture medium without fetal bovine serum was added into the upper chamber for $24 \mathrm{~h} 600 \mu \mathrm{l}$ RPMI 1640 medium containing $20 \%$ fetal bovine serum which was added in the lower chamber simultaneously. After $24 \mathrm{~h}$, the nonmigrating cells on the top of Transwell ependyma were removed with a cotton swab. The cells were fixed with $1 \%$ paraformaldehyde at room temperature for $30 \mathrm{~min}$ and stained with $2 \%$ crystal violet for $30 \mathrm{~min}$. The transmembrane cells on the ventricular membrane were imaged with an inverted microscope.

\section{Transwell Method was Used to Detect the Invasive Activity of Cells}

The cells were inoculated into the Transwell upper chamber (Corning, USA) with Matrigel glue at $2 \times 10^{5} /$ well density. The cells in the upper chamber were cultured with a corresponding culture medium without fetal bovine serum for $24 \mathrm{~h}$ with $600 \mu \mathrm{l}$ RPMI 1640 medium containing 20\% fetal bovine serum in the lower chamber simultaneously. After $24 \mathrm{~h}$, the nonmigrating cells on the top of Transwell ependyma were removed with a cotton swab. After $1 \%$ paraformaldehyde was fixed at room temperature for $30 \mathrm{~min}$ and stained with $2 \%$ crystal violet for $30 \mathrm{~min}$, the transmembrane cells on the ventricular membrane in 3 randomly selected fields of each group were imaged with an inverted microscope to count the cell number.

\section{Flow Cytometry was Utilized to Measure Apoptosis}

Apoptosis was determined by Annexin V-FITC/PI double staining. The cells were divided into control and SPUP treatment groups, inoculated in 6-well plates at the density of $10^{5} /$ well, and treated the next day. After $24 \mathrm{~h}$ of culture, the cells were trypsinized and transferred to the centrifuge tube and washed with precooled PBS 3 times with centrifuging $5 \mathrm{~min}$ each. After cell pellets were obtained, $500 \mu$ l combined buffer was added and incubated in the dark, in which $5 \mu$ l Annexin V-FITC was added with $10 \mu \mathrm{l}$ PI staining solution. The cells were cultured in the dark at room temperature for $15 \mathrm{~min}$. Then, flow cytometry was performed and analyzed using FlowJo software to evaluate cell apoptosis.

\section{Western Blot was Used to Detect the Protein Expression of Cells}

RIPA (Beyotime Technology, China) containing PMSF and phosphotransferase inhibitor was used for cell protein extraction. The BCA protein concentration analysis kit (Beyotime Technology, Shanghai, China) was used to determine the protein concentration. An equal amount of proteins was separated using SDS-PAGE gel (12\%) and transferred to the PVDF membrane (Millipore, Bedford, MA, USA). Subsequently, the membrane was blocked with $5 \%$ skimmed milk at room temperature for $1 \mathrm{~h}$ and incubated with the corresponding primary antibody at $4^{\circ} \mathrm{C}$ overnight. The horseradish peroxidase bound secondary antibody was used to incubate at room temperature for $1 \mathrm{~h}$. Protein fluorescence imaging was performed in the developing system using an ECL luminescent solution (Millipore, USA). Primary reactance includes $\beta$-actin (1:3,000, ab8226, Abcam, United Sates), Shh (1,000, ab53281, Abcam), Gli1 (1:1,000, ab134906, Abcam), Ptch1 (1:500, ab53715, Abcam), and Smo (1:1,000, ab72130, Abcam).

\section{Statistical Analysis}

All experiments were repeated three times independently. All measurement data in this study were expressed as mean \pm standard deviation. SPSS 20.2 (SPSS, Chicago, IL, USA) was used. Under the same conditions, one-way ANOVA was used for intragroup comparison. Different groups were compared using Student's t-test. When $p<0.05$, the difference was significant.

\section{RESULTS}

\section{Identification of Physical and Chemical Properties of SPUP}

Through chemical analysis, the main contents of SPUP were identified as total sugar (80.21\%), uronic acid (3.56\%), and protein $(7.32 \%)$. Notably, the percentage of sulfate in SPUP was $29.47 \%$, suggesting that SPUP was an acid-free polysaccharide. As shown in Figure 1A, consistent with chemical composition analysis, the UV-Vis spectrum of SPUP reported an obvious absorption at almost $280 \mathrm{~nm}$, indicating the existence of protein or nucleic acid in SPUP (Feng et al., 2010; Zhao et al., 2018).

Following, the monosaccharide composition of SPUP was analyzed and cross referenced with the chromatogram of monosaccharide standard. As shown in Figure 1B, monosaccharide standards of rhamnose, arabinose, fucose, xylose, mannose, glucose, and galactose were separated well under GC conditions. In contrast, SPUP was mainly composed of fucose, glucose, and galactose, with a molar ratio of 27.22:19.32: 53.46 (Figure 1C).

In addition, the bond structure of SPUP was determined by infrared spectroscopy. Similar to common carbohydrate features, SPUP exhibited a stretching vibration of the $\mathrm{O}-\mathrm{H}$ bond at 3,437.1 cm-1 (Figure 1D). A stretching vibration of the $\mathrm{C}-\mathrm{H}$ key could then be seen at $2,981.3 \mathrm{~cm}-1$. The moderateintensity absorption peaks appeared at 1,661.2 and 1,638.7 cm1 , manifested explicitly in the deformation vibration of the $\mathrm{O}-\mathrm{H}$ bond. There was a strong absorption peak at about $1,000-1,200 \mathrm{~cm}^{-1}$, suggesting the stretching of the coupled 
A

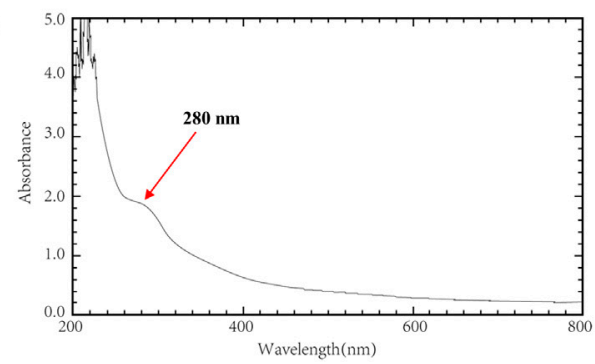

C

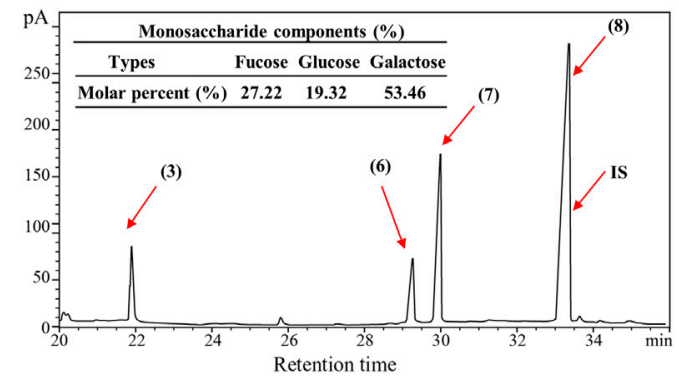

E

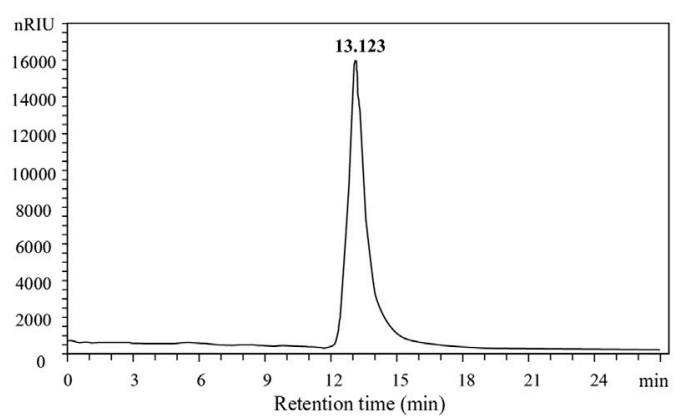

B

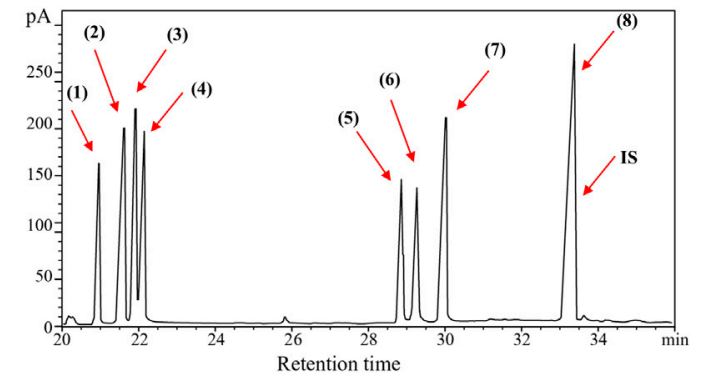

D

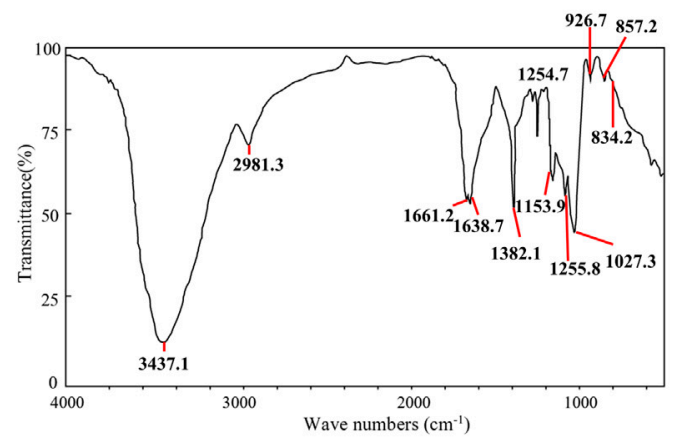

FIGURE 1 | Identification of physical and chemical properties of SPUP; (A) UV-vis spectra of SPUP; (B,C) GC diagram of standard monosaccharide and SPUP; (D) infrared spectrum of SPUP; (E) HPGPC chromatogram of SPUP. Note: 1): rhamnose; 2): arabinose; 3): fucose; 4): xylose; 5): mannose; 6): glucose; 7): galactose; 8): inositol; IS: internal standard. SPUP inhibited the proliferation of OC cells.

A HOSEPICs

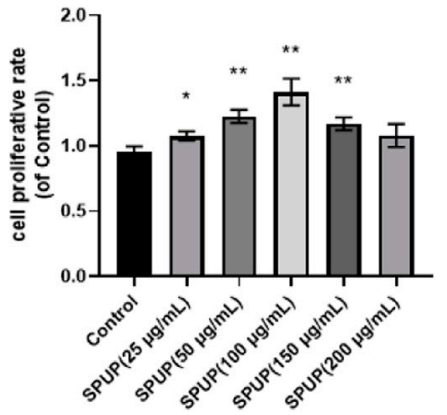

B

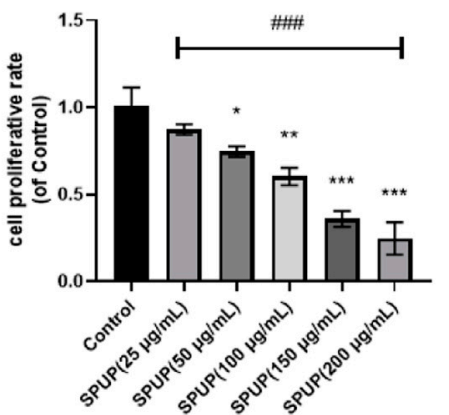

C

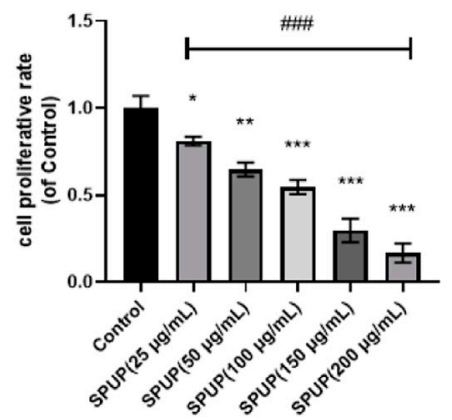

FIGURE 2 | Effect of SPUP on the proliferation activity of two kinds of OC cells and human normal ovarian epithelial cells. Compared with the control group, ${ }^{*} p<$

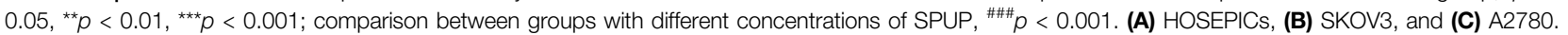




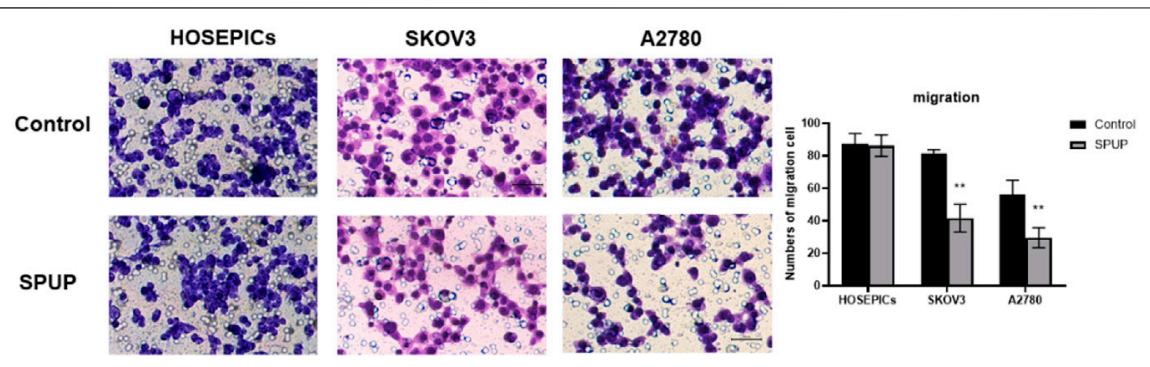

FIGURE 3 | Effect of SPUP on the migration ability of two kinds of OC cells and human normal ovarian epithelial cells. Compared with the control group, ${ }^{* \star} p<0.01$.

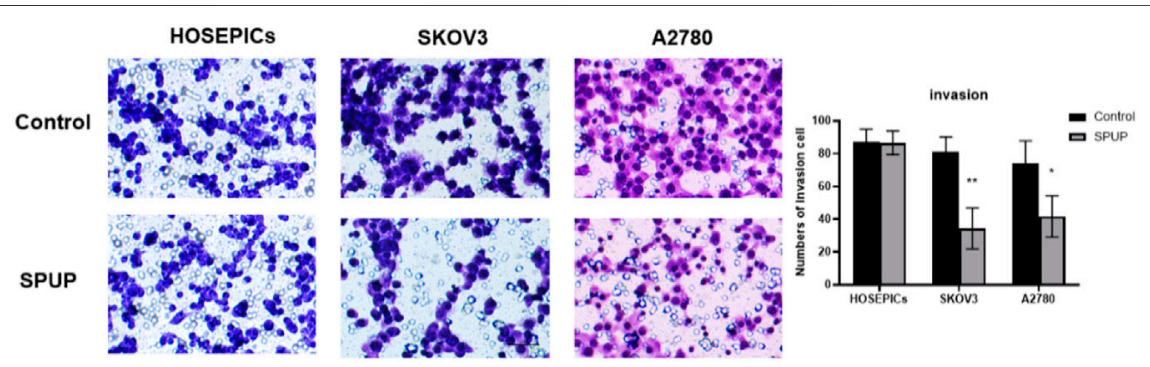

FIGURE 4 |SPUP on the invasion ability of two kinds of OC cells and human normal ovarian epithelial cells. Compared with the control group, ${ }^{*} p<0.05,{ }^{* *} p<0.01$.

$\mathrm{C}-\mathrm{O}$ bond and $\mathrm{C}-\mathrm{C}$ bond, as well as the bending vibration of $\mathrm{O}-\mathrm{H}$. The absorption peak signal at $926.7 \mathrm{~cm}^{-1}$ might be due to the presence of $\beta$-d-galactopyranose. In addition, according to previous studies, it could be determined that the absorption peak at 834.2 or 857.2 and $1,255.8 \mathrm{~cm}^{-1}$ was sulfate (Mao et al., 2008). In addition, the characteristic absorption, peaking at 834.2 or 857.2 , suggested the existence of an a-type glycosidic bond in SPUP.

As shown in Figure 1E, the retention time of SPUP in HPGPC chromatography was $13.123 \mathrm{~min}$. According to the calibration curve-fitting equation established by the dextran standard with different molecular weights, the calculation method of retention time was substituted into the calibration curve equation. The average molecular weight of SPUP was $97.9 \mathrm{kDa}$.

\section{SPUP Inhibited the Proliferation of OC Cells}

Through the proliferation assay analysis, with the increase in SPUP concentration, the proliferative activity of two OC cells decreased in a concentration-dependent manner $(p<0.05)$ (Figure 2). The cell proliferation rate was decreased under 0.5 when the concentration of SPUP reached $150 \mu \mathrm{g} / \mathrm{ml}$. In contrast, in human normal ovarian epithelial cells, the capacity of cell proliferation increased with SPUP treatment within the range of $25-100 \mu \mathrm{g} / \mathrm{ml}(p<0.05)$. However, the increase in cell proliferation rate caused by SPUP reached its maximum at $100 \mu \mathrm{g} / \mathrm{ml}$ and no additional effects were observed with further increasing of SPUP. Taken from the results, SPUP could inhibit the proliferation of OC cells in a dosage-dependent manner. As determined, the concentrations of 150 and $200 \mu \mathrm{g} /$ $\mathrm{ml}$ of SPUP inhibited cell proliferation most violently; the concentration of $100 \mu \mathrm{g} / \mathrm{ml}$ SPUP was used for further study otherwise indicated.

\section{SPUP Reduced OC Cell Migration}

To study the effect of SPUP on the migration ability of HOSEPICs, SKOV3, and A2780 cells, Transwell cell migration experiments were used to determine the migration ability of cells. As shown in Figure 3, SPUP treatment could significantly inhibit the migration ability of OC cells (all $p<$ $0.05)$ than the control group. In contrast, there are no significant changes observed in the migration ability of normal cells $(p>0.05)$.

\section{SPUP Treatment Inhibited OC Cell Invasion} The invasion ability of OC cells was measured by the Transwell chamber. Compared with the control group, SPUP treatment could significantly inhibit the invasion ability of OC cells (all $p<$ 0.05 ) (Figure 4). In comparison, the migration ability of normal cells did not change significantly $(p>0.05)$.

\section{SPUP Promoted OC Cell Apoptosis}

As shown in Figure 5, SPUP treatment could significantly induce the apoptosis of OC cells (all $p<0.05$ ) but not in normal cells $(p>0.05)$.

\section{SPUP Inhibited the Activation of Hedgehog Signaling Pathway in OC Cells}

As shown in Figure 6A, OC cells had different degrees of $\mathrm{Hh}$ signal pathway activation compared with HOSEPICs cells (all $p<0.05)$. To clarify whether SPUP plays an anti-OC role by 

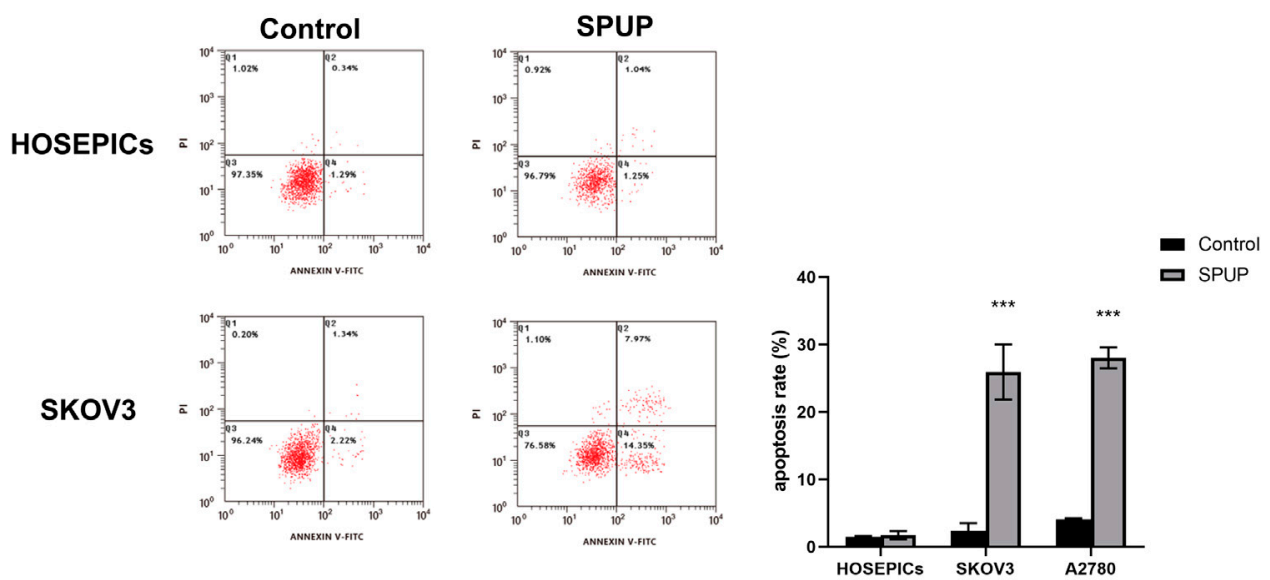

A2780
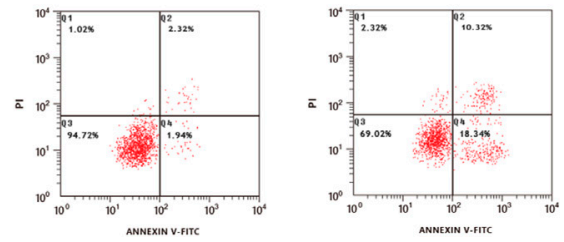

FIGURE 5 | Effects of SPUP on apoptosis of two kinds of OC cells and human normal ovarian epithelial cells. ${ }^{* \star *} p<0.001$ compared with the control group. SPUP inhibited the activation of the hedgehog signaling pathway in OC cells.

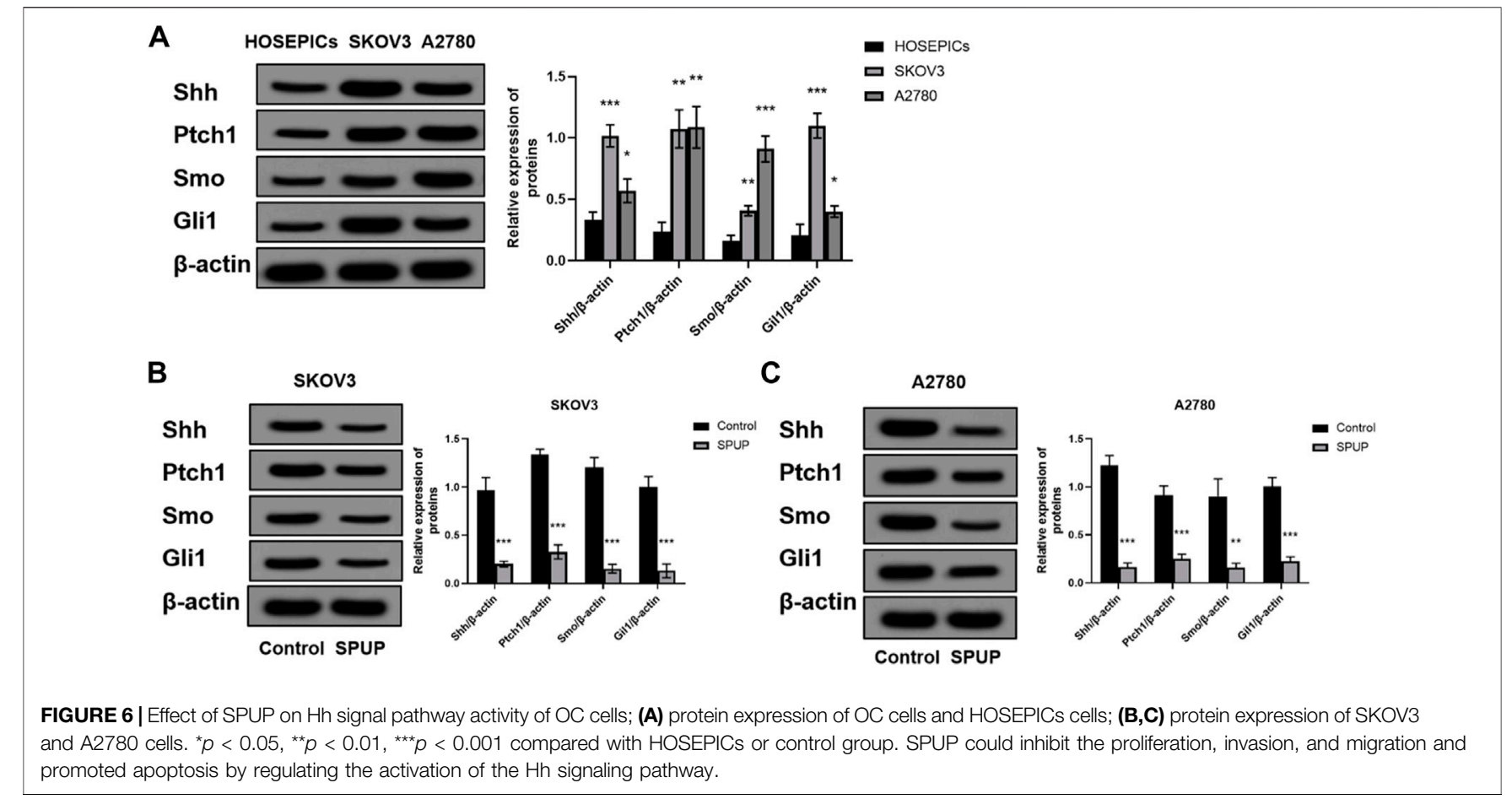

inhibiting the Hh pathway, we evaluated the expressions of Shh, Ptch1, Smo, and Gli1 upon SPUP treatment. As shown in Figures 6B,C, compared with the control group, the expression of Shh, Ptch1, Smo, and Gli1 proteins in OC cells in the SPUP treatment group decreased to varying degrees (all $p<0.05)$. The results suggested that SPUP might take effects in anticancer through inhibiting the activation of the Hh signal pathway in OC cells. 

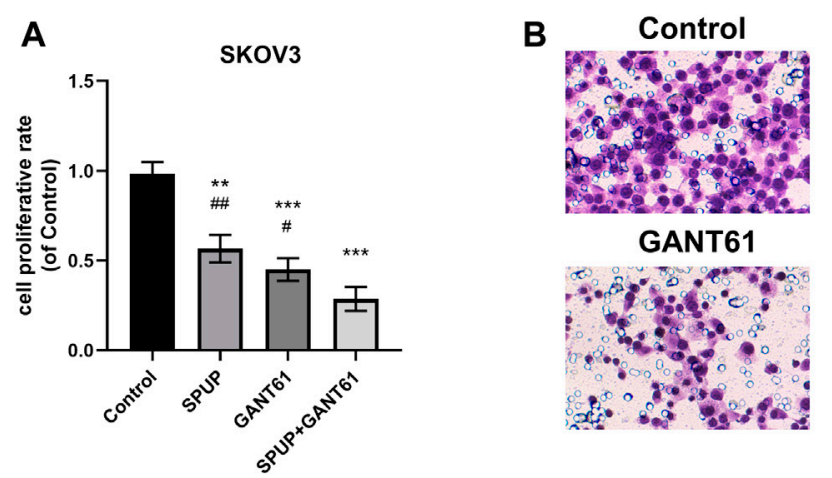

GANT61
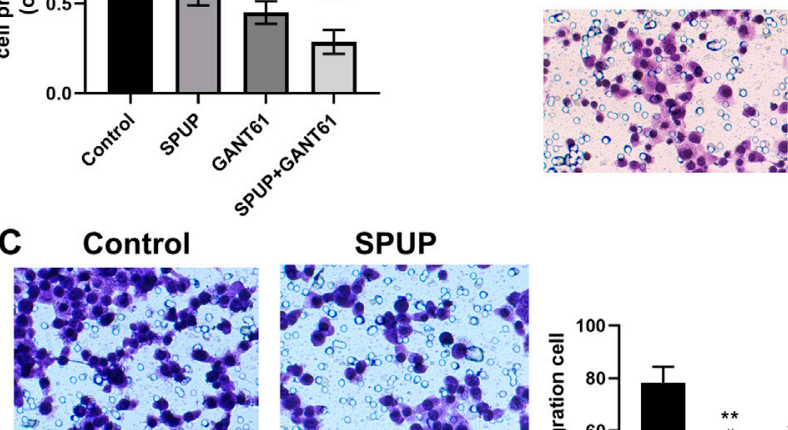

GANT61

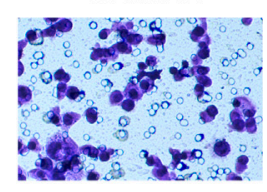

D

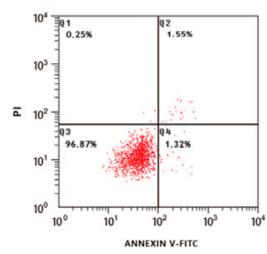

Control
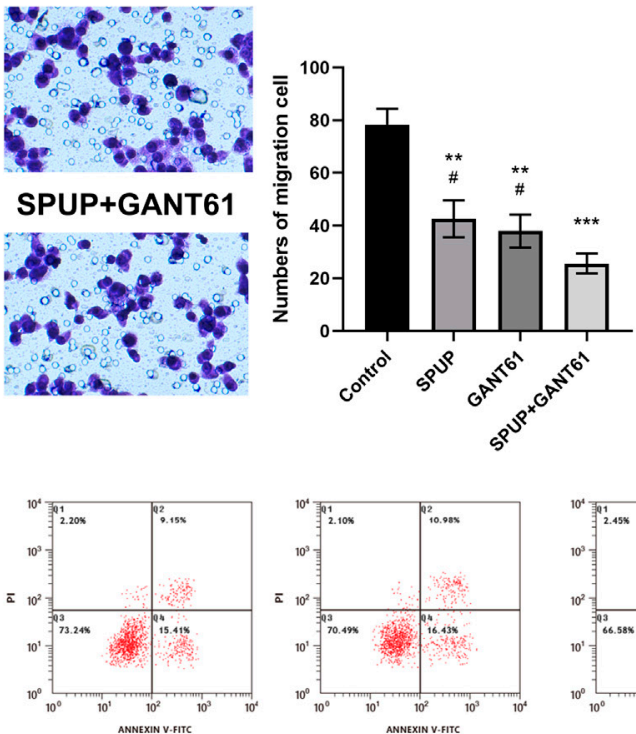

SPUP

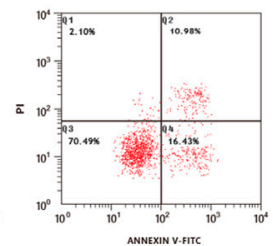

GANT61

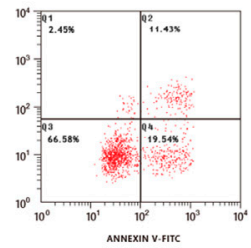

SPUP+GANT61
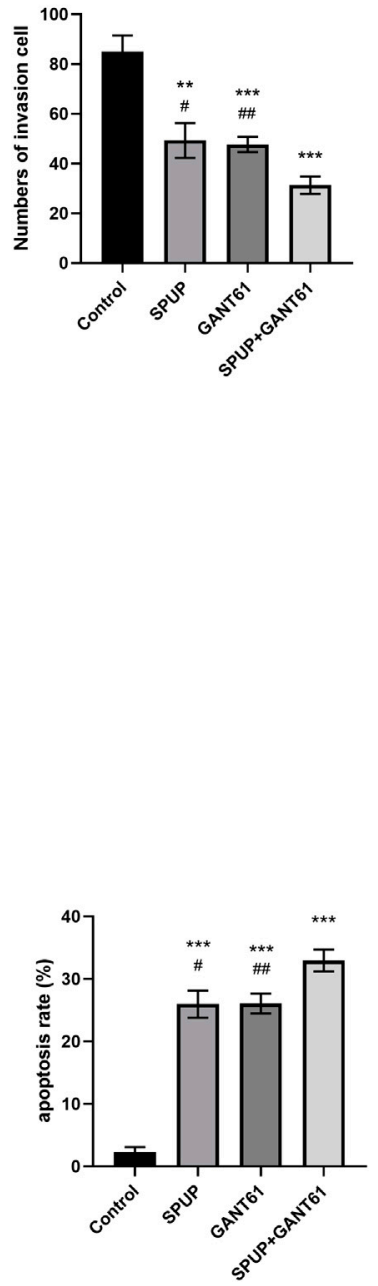

FIGURE 7 |SPUP plays a role in regulating cell function by inhibiting the activation of the $\mathrm{Hh}$ signaling pathway. (A) The CCK-8 method was used to determine the proliferation ability of cells after the Hh pathway inhibitor was applied; (B,C) the Transwell method was used to determine the invasion and migration ability of cells after Hh pathway inhibitor was applied; (D) the apoptosis of cells after the Hh pathway inhibitor was measured by flow cytometry. ${ }^{* *} p<0.01,{ }^{* \star *} p<0.001$ compared with the control group, ${ }^{\#} p<0.01,{ }^{\# \#} p<0.01$ compared with the SPUP + GANT61 group.

\section{SPUP Could Inhibit Proliferation, Invasion, and Migration and Promote Apoptosis by Regulating the Activation of the Hh Signaling Pathway}

As shown in Figure 7, SPUP or GANT61 alone or in combination with SPUP and GANT61 could inhibit the proliferation, migration, and invasion of OC cells (all $p<0.05$ ) and promote apoptosis (all $p<0.05$ ) in SKOV3 cells. The inhibitory effects of the SPUP + GANT61 group were more obvious than those of the SPUP or GANT61 group (all $p<0.05$ ), respectively. Thus, SPUP played an anticancer role by regulating the activation of the $\mathrm{Hh}$ signaling pathway.

\section{DISCUSSION}

As known, SPUP is an effective apoptosis inducer for a variety of cancer cells (Yang et al., 2013). In addition to anticancer, antiproliferative, and anticoagulant activities, some studies have also found that SPUP can activate exogenous or endogenous apoptosis pathways in various cancer cell lines through regulating the expression of apoptosis-related or signal proteins, cell-cycle regulatory proteins, and transcription factors (Hyun et al., 2009; Yamasaki-Miyamoto et al., 2009). This study found that SPUP could inhibit the proliferation, migration, and invasion of OC cells and induce apoptosis. In addition, SPUP significantly reduced the activation of the Hh signaling pathway at the protein level. 
As previous study has shown that SPUP could inhibit the proliferation and migration and induce the apoptosis of breast cancer cells (Wu et al., 2019), we thought to investigate the role of SPUP in OC cells. A systematic evaluation of cell proliferation, migration, invasion, and apoptosis was conducted using a combination of CCK- 8 assay, Transwell assay, and flow cytometry. We, for the first time, confirmed that SPUP could inhibit the cell proliferation of OC cells (SKOV3 and A2780) in a dose-dependent manner. The concentrations of SPUP $(25,50$, 100,150 , and $200 \mu \mathrm{g} / \mathrm{ml}$ ) we used in this study are consistent with Wu et al. (Wu et al., 2019), who once found that SPUP treatment could inhibit the proliferation and migration and induce the apoptosis of breast cancer cells. Considering that there are few studies that focus on the effects of SPUP in cancers, more experimental studies are still needed.

Moreover, this study also provided novel insights in the effects of SPUP on normal human ovarian epithelial cells. It was found that SPUP could promote the proliferation of human ovarian epithelial cells, suggesting that the side effects of SPUP treatment could be reduced to the minimum by administering a proper amount of SPUP. Migration and invasion are known to play an essential role in the progression of tumors. Our study also found for the first time that SPUP could significantly inhibit the migration and invasion of OC cells and induce the apoptosis of SPUP-treated OC cells.

The Hh pathway is very important for embryonic development (Wang et al., 2017). Many previous studies have shown that activation of the Hh pathway could promote the occurrence and development of tumors, including OC (Chen et al., 2013; $\mathrm{Wu}$ et al., 2019). The Hh pathway is known to have a high expression level in OC (Schmid et al., 2011). Taken together, inhibition of Hh signals could induce apoptosis and inhibit cell viability and migration. Nevertheless, inhibition of the Hh pathway may be an effective target for OC treatment. Corresponding to previous studies, this study also investigated the relationship between SPUP and the Hh signaling pathway. In our study, we compared the expression of the $\mathrm{Hh}$ signaling pathway-related proteins in human normal ovarian epithelial cells and OC cells and found that the levels of Shh, Ptch1, Smo, and Gli1 protein in OC cells were significantly increased, indicating that the $\mathrm{Hh}$ signaling pathway was activated in OC. Moreover, we were the first time to find that the activation of the Hh signaling pathway was diminished upon SPUP treatment in SKOV3 and A2780 cells. Therefore, we speculated that SPUP might play an anticancer role in OC by inhibiting Hh signal activation. Till now, there are few studies that reported the regulatory effects of polysaccharide on the Hh signaling pathway in cancers. Yamasaki et al. once found that

\section{REFERENCES}

Chen, Q., Gao, G., and Luo, S. (2013). Hedgehog Signaling Pathway and Ovarian Cancer. Chin. J. Cancer Res. 25 (3), 346-353. doi:10.3978/j.issn.10009604.2013.06.04

Chien, J., Kuang, R., Landen, C., and Shridhar, V. (2013). Platinum-sensitive Recurrence in Ovarian Cancer: the Role of Tumor Microenvironment. Front. Oncol. 3, 251. doi:10.3389/fonc.2013.00251 protein-bound polysaccharide- $\mathrm{K}$ could inhibit the induction of a malignant phenotype in pancreatic cancer by suppressing the $\mathrm{Hh}$ signaling pathway (Yamasaki et al., 2016), suggesting that polysaccharide might suppress the progression of cancers by modulating the activation of Hh signaling. In our study, we also found similar results; however, the underlying mechanism effects of SPUP in OC still needs further research.

In this study, SKOV3 cells were used as a disease model and treated with SPUP, GANT61 (Hh pathway inhibitor), and SPUP and GANT61 to investigate the underlying mechanism of SPUP on the Hh signal pathway. We found that SPUP could induce apoptosis of OC cells and inhibit cell proliferation, migration, and invasion. The effect of the GANT61 treatment was similar to that of SPUP. In addition, the anticancer effect of SPUP combined with GANT61 was more efficient than that of GANT61 alone, suggesting that SPUP could enhance the anticancer effects of GANT61. The above results indicated that SPUP might induce OC cell apoptosis and inhibit cell proliferation, migration, and invasion by reducing the protein expression of Hh signal pathway proteins Shh, Ptch1, Smo, and Gli1, similar to other Hh signal pathway inhibitors.

In conclusion, this study identified SPUP, for the first time, as an effective drug which induced apoptosis and inhibited cell proliferation, migration, and invasion by inhibiting Hh pathway conduction in OC cells. However, the exact mechanism of how SPUP regulating Hh pathway in OC was still in need for further studies.

\section{DATA AVAILABILITY STATEMENT}

The original contributions presented in the study are included in the article/Supplementary Material; further inquiries can be directed to the corresponding author.

\section{AUTHOR CONTRIBUTIONS}

YY and YQ proposed and designed the experiments. YY and QZ carried out the experiments with the help of YX and GC. YY and QZ drafted the article and interpreted the data. GC, YX, and YQ revised the article. All the authors approved the final version of this article.

\section{FUNDING}

The project was supported by the Jiaxing Science and Technology Plan Project (No. 2018AD32182).

Chowdhury, S. R., Sengupta, S., Biswas, S., Sen, R., Sinha, T. K., Basak, R. K., et al (2015). Low Fucose Containing Bacterial Polysaccharide Facilitate Mitochondriadependent ROS-Induced Apoptosis of Human Lung Epithelial Carcinoma via Controlled Regulation of MAPKs-Mediated Nrf2/Keap1 Homeostasis Signaling. Mol. Carcinog. 54 (12), 1636-1655. doi:10.1002/mc.22236

Feng, L., Jia, X.-B., Shi, F., and Chen, Y. (2010). Identification of Two Polysaccharides from Prunella Vulgaris L. And Evaluation on Their Antilung Adenocarcinoma Activity. Molecules 15 (8), 5093-5103. doi:10.3390/ molecules15085093 
Ferlay, J., Soerjomataram, I., Dikshit, R., Eser, S., Mathers, C., Rebelo, M., et al. (2015). Cancer Incidence and Mortality Worldwide: Sources, Methods and Major Patterns in GLOBOCAN 2012. Int. J. Cancer 136 (5), E359-E386. doi:10.1002/ijc. 29210

Fu, X., Li, Y., Alvero, A., Li, J., Wu, Q., Xiao, Q., et al. (2016). MicroRNA-222-3p/ GNAI2/AKT axis Inhibits Epithelial Ovarian Cancer Cell Growth and Associates with Good Overall Survival. Oncotarget 7 (49), 80633-80654. doi:10.18632/oncotarget.13017

Hyun, J.-H., Kim, S.-C., Kang, J.-I., Kim, M.-K., Boo, H.-J., Kwon, J.-M., et al. (2009). Apoptosis Inducing Activity of Fucoidan in HCT-15 colon Carcinoma Cells. Biol. Pharm. Bull. 32 (10), 1760-1764. doi:10.1248/bpb.32.1760

Liu, Y., Gao, S., Zhu, J., Zheng, Y., Zhang, H., and Sun, H. (2018). Dihydroartemisinin Induces Apoptosis and Inhibits Proliferation, Migration, and Invasion in Epithelial Ovarian Cancer via Inhibition of the Hedgehog Signaling Pathway. Cancer Med. 7 (11), 5704-5715. doi:10.1002/cam4.1827

Mao, W.-J., Fang, F., Li, H.-Y., Qi, X.-H., Sun, H.-H., Chen, Y., et al. (2008). Heparinoid-active Two Sulfated Polysaccharides Isolated from marine green Algae Monostroma Nitidum. Carbohydr. Polym. 74 (4), 834-839. doi:10.1016/ j.carbpol.2008.04.041

Mutch, D. G., and Prat, J. (2014). 2014 FIGO Staging for Ovarian, Fallopian Tube and Peritoneal Cancer. Gynecol. Oncol. 133 (3), 401-404. doi:10.1016/ j.ygyno.2014.04.013

Reid, B. M., Permuth, J. B., and Sellers, T. A. (2017). Epidemiology of Ovarian Cancer: a Review. Cancer Biol. Med. 14 (1), 9-32. doi:10.20892/j.issn.20953941.2016.0084

Schmid, S., Bieber, M., Zhang, F., Zhang, M., He, B., Jablons, D., et al. (2011). Wnt and Hedgehog Gene Pathway Expression in Serous Ovarian Cancer. Int. J. Gynecol. Cancer 21 (6), 975-980. doi:10.1097/igc.0b013e31821caa6f

Sevag, M. G., Lackman, D. B., and Smolens, J. (1938). The Isolation of the Components of Streptococcal Nucleoproteins in Serologically Active Form. J. Biol. Chem. 124 (1), 42-49. doi:10.1016/s0021-9258(18)74048-9

Skoda, A. M., Simovic, D., Karin, V., Kardum, V., Vranic, S., and Serman, L. (2018). The Role of the Hedgehog Signaling Pathway in Cancer: A Comprehensive Review. Bosn J. Basic Med. Sci. 18 (1), 8-20. doi:10.17305/bjbms.2018.2756

Song, Z., Li, H., Liang, J., Xu, Y., Zhu, L., Ye, X., et al. (2019). Sulfated Polysaccharide from Undaria Pinnatifida Stabilizes the Atherosclerotic Plaque via Enhancing the Dominance of the Stabilizing Components. Int. J. Biol. Macromolecules 140, 621-630. doi:10.1016/j.ijbiomac.2019.08.173

Torre, L. A., Trabert, B., DeSantis, C. E., Miller, K. D., Samimi, G., Runowicz, C. D., et al. (2018). Ovarian Cancer Statistics, 2018. CA: A Cancer J. Clinicians 68 (4), 284-296. doi:10.3322/caac.21456

Wang, X., Wei, S., Zhao, Y., Shi, C., Liu, P., Zhang, C., et al. (2017). Antiproliferation of Breast Cancer Cells with Itraconazole: Hedgehog Pathway Inhibition Induces Apoptosis and Autophagic Cell Death. Cancer Lett. 385, 128-136. doi:10.1016/j.canlet.2016.10.034

Wu, J., Li, H., Wang, X., Zhang, X., Liu, W., Wang, Y., et al. (2019). Effect of Polysaccharide from Undaria Pinnatifida on Proliferation, Migration and Apoptosis of Breast Cancer Cell MCF7. Int. J. Biol. Macromolecules 121, 734-742. doi:10.1016/j.ijbiomac.2018.10.086
Yamasaki, A., Onishi, H., Imaizumi, A., Kawamoto, M., Fujimura, A., Oyama, Y., et al. (2016). Protein-bound Polysaccharide-K Inhibits Hedgehog Signaling through Down-Regulation of MAML3 and RBPJ Transcription under Hypoxia, Suppressing the Malignant Phenotype in Pancreatic Cancer. Anticancer Res. 36 (8), 3945-3952.

Yamasaki-Miyamoto, Y., Yamasaki, M., Tachibana, H., and Yamada, K. (2009). Fucoidan Induces Apoptosis through Activation of Caspase- 8 on Human Breast Cancer MCF-7 Cells. J. Agric. Food Chem. 57 (18), 8677-8682. doi:10.1021/jf9010406

Yang, L., Wang, P., Wang, H., Li, Q., Teng, H., Liu, Z., et al. (2013). Fucoidan Derived from Undaria Pinnatifida Induces Apoptosis in Human Hepatocellular Carcinoma SMMC-7721 Cells via the ROS-Mediated Mitochondrial Pathway. Mar. Drugs 11 (6), 1961-1976. doi:10.3390/ md11061961

Yu, Y., Zhang, Y., Hu, C., Zou, X., Lin, Y., Xia, Y., et al. (2019). Chemistry and Immunostimulatory Activity of a Polysaccharide from Undaria Pinnatifida. Food Chem. Toxicol. 128, 119-128. doi:10.1016/j.fct.2019.03.042

Zhao, Y., Zheng, Y., Wang, J., Ma, S., Yu, Y., White, W., et al. (2018). Fucoidan Extracted from Undaria Pinnatifida: Source for Nutraceuticals/Functional Foods. Mar. Drugs 16 (9), 321. doi:10.3390/md16090321

Zhou, A. Y., Robertson, J., Hamid, N., Ma, Q., and Lu, J. (2015). Changes in Total Nitrogen and Amino Acid Composition of New Zealand Undaria Pinnatifida with Growth, Location and Plant Parts. Food Chem. 186, 319-325. doi:10.1016/ j.foodchem.2014.06.016

Zong, A., Cao, H., and Wang, F. (2012). Anticancer Polysaccharides from Natural Resources: a Review of Recent Research. Carbohydr. Polym. 90 (4), 1395-1410. doi:10.1016/j.carbpol.2012.07.026

Zong, A., Liu, Y., Zhang, Y., Song, X., Shi, Y., Cao, H., et al. (2015). Anti-tumor Activity and the Mechanism of SIP-S: A Sulfated Polysaccharide with Anti-metastatic Effect. Carbohydr. Polym. 129, 50-54. doi:10.1016/ j.carbpol.2015.04.017

Conflict of Interest: The authors declare that the research was conducted in the absence of any commercial or financial relationships that could be construed as a potential conflict of interest.

Publisher's Note: All claims expressed in this article are solely those of the authors and do not necessarily represent those of their affiliated organizations, or those of the publisher, the editors, and the reviewers. Any product that may be evaluated in this article, or claim that may be made by its manufacturer, is not guaranteed or endorsed by the publisher.

Copyright (C) 2021 Yang, Zhang, Xu, Chen and Qiu. This is an open-access article distributed under the terms of the Creative Commons Attribution License (CC BY). The use, distribution or reproduction in other forums is permitted, provided the original author(s) and the copyright owner(s) are credited and that the original publication in this journal is cited, in accordance with accepted academic practice. No use, distribution or reproduction is permitted which does not comply with these terms. 